\title{
Inducing tunable single-mode operation of Fabry-Perot semiconductor laser coupled with very-short cavity with selective optical feedback
}

\author{
Mohammed S. Al-Ghamdi ${ }^{1}$, Ahmed Bakry ${ }^{1}$ and Moustafa Ahmed ${ }^{1,2^{*}}$
}

\begin{abstract}
We report on achieving tuned single mode operation of a multimode semiconductor laser by coupling it to a veryshort external cavity with selective feedback. We show that the induced uniform feedback light from the very-short external cavity can induce single-mode oscillation over a broad range of optical feedback. Then by applying selective external to the optimum settings of the single-mode oscillation, we predict tuning of the single-mode output over a wavelength range of $\sim 20 \mathrm{~nm}$ by detuning the peak wavelength of optical feedback from the central wavelength of the modal gain. The study is based on modeling of the dynamics of multimode semiconductor laser subject to selective optical feedback. The model takes into account mechanisms of both self- and cross-modal gain suppression, and the study is concerned with the very-short external cavity that corresponds to stable continuouswave $(\mathrm{CW})$ operation.
\end{abstract}

Keywords: Multimode laser, Optical feedback, Optical gain, Semiconductor laser

\section{Introduction}

Subjecting semiconductor laser to external optical feedback induces variety of changes in the laser output, ranging between linewidth narrowing to coherence collapse, and from stable $\mathrm{CW}$ operation or period-1 oscillations with low noise to chaotic dynamics with noisiest levels [1-15]. An experimental study of the so-called 'regimes of feedback' and the associated characteristic dynamics of semiconductor lasers was undertaken by Tkach and Chaprylyvy [15]. The distance between the laser facet and the external reflector plays a significant role in determining the nature of the response of semiconductor lasers to optical feedback as pointed out in $[9,13,16]$. When the external cavity formed between the laser front facet and external reflector is long enough, the laser output is mainly chaotic and could exhibit low-frequency fluctuations [17]. As the length decreases, the laser

\footnotetext{
* Correspondence: mostafa.farghal@mu.edu.eg

${ }^{1}$ Department of Physics, Faculty of Science, King Abdulaziz University, 80203, Jeddah 21589, Saudi Arabia

2Department of Physics, Faculty of Science, Minia University, Egypt, Minia 61519, Egypt
}

\section{Springer Open}

(c) The Author(s). 2019 Open Access This article is distributed under the terms of the Creative Commons Attribution 4.0 International License (http://creativecommons.org/licenses/by/4.0/), which permits unrestricted use, distribution, and reproduction in any medium, provided you give appropriate credit to the original author(s) and the source, provide a link to the Creative Commons license, and indicate if changes were made.

exhibits chaos cycles with the increase of the feedback strength. In this case the route-to-chaos may include period-one oscillations, period doubling, or subharmonics oscillations depending on the period of the round-trip in the external cavity relative to the period of relaxation oscillation of the laser $[9,12,18,19]$. As the external cavity gets shorter, the number of chaos cycles decreases and the feedback range of the chaos cycle becomes narrower [20]. In the limit of the very short cavity, the chaos cycles may disappear and the laser operation becomes more stable [20]. This limit is also advantageous to enhance the modulation bandwidth and speed of the laser due to the induced photon-photonresonance effect between the modulating field and the external-cavity oscillating modes [21].

On the other hand, although semiconductor laser with the Fabry-Perot cavity type is preferential in costeffective optical systems, its emission spectral width can be quite broad $(\sim 1 \mathrm{THz})$. Therefore this type of semiconductor laser supports oscillations of a large number of coupled longitudinal modes. In principle, when the laser design is optimized in the transverse direction, 
single mode oscillation could be possible. This singlemode oscillation was observed in AlGaAs lasers when the injection current is well-above the threshold level [22]. In lasers made from the quaternary compound InGaAsP, the gain is rather shallow and spectrally asymmetric, which supports oscillation of several modes on the long-wavelength side of the central mode [23-26]. It was observed in experiments that these modes exhibit hopping, which was attributed to the large value of the linewidth enhancement factor that violates the asymmetric gain suppression (AGS) and competition among these long-wavelength modes [27]. It was shown that this multimode hopping can be released and the laser supports single-mode oscillation by subjecting the laser to very strong optical feedback and avoiding the chaotic state $[28,29]$. They pointed out that the range of feedback that corresponds to single-mode oscillation shifts to weaker values with shortening the external cavity [28]. It is then motivating to investigate the possibility of achieving stable single-mode oscillation under the predicted stable operation of semiconductor laser coupled with a very-short external cavity. Introducing selective feedback to lock or tune the predicted single-mode oscillation could add to the advantages of this coupled laser as a promising device in wavelength-division multiplexing transmission systems [30], and optical pumping [31]. The selective feedback can be achieved using diffraction gratings [32] or tunable wavelength selective grating structures, such as grating waveguide structures [33] and resonant grating mirrors [34].

In this paper, we investigate the modal behavior of multimode semiconductor laser subject to optical feedback from a very-closed reflector, aiming at allocating the range of feedback strength that induces stable single-mode oscillation. We also examine the possibility of achieving tunable single mode oscillation by using spectrally selective optical feedback. First, we used a spectrally uniform optical feedback from a very short external optical feedback to convert the multimode oscillation of the laser into single-mode oscillation over a wide range of optical feedback. Then we apply the selective external reflectivity to the optimum settings of stable single-mode oscillation to tune this single mode output. The single-mode output is tuned by detuning the mode wavelength with respect with the peaked wavelength of the external reflectivity. The study is based on numerical integration of time-delay rate equations of multimode semiconductor laser under selective optical feedback. The feedback is included as time delay of the laser modes due to multiple round trips in the external cavity. The model takes into account both mechanisms of self- and cross-modal suppressions of modal gain, with the latter effect including the spectral symmetric and asymmetric gain suppressions. Because
AGS is strong in this case of long-wavelength laser, we compare the obtained results with those obtained using homogeneous gain broadening (HGB) to differentiate between the influence of optical feedback and AGS. The results show that the feedback from the very-closed reflector converts the multimode oscillation into singlemode oscillation over a wide range of feedback covering the weak feedback regime. The spectrally selective feedback is predicted to tune this single mode oscillation over a wavelength range as wide as $20 \mathrm{~nm}$.

\section{Simulation model}

In semiconductor lasers, coupling among the longitudinal oscillating modes is introduced through the optical gain $G_{p}$, which includes two terms

$$
G_{p}=G_{L(p)}-G_{N L(p)}
$$

The first term $G_{L(p)}$ represents linear gain, which is proportional to the injected carrier number $N$ and has parabolic spectral broadening [26].

$$
G_{L(p)}=\frac{a \xi}{V}\left[N-N_{g}-b V\left(\lambda_{p}-\lambda_{\text {peak }}\right)^{2}\right] \quad p=0, \pm 1, \pm 2, \ldots
$$

where $a$ is the tangent gain coefficient, $N_{g}$ is the transparency level of the carrier number, and $b$ is the spectrum width. $V$ is the volume of the active region whose length is $L_{D}$ and refractive index is $n_{D}$. $\lambda_{\text {peak }}$ is the wavelength at the peak of the spectrum. The central mode $p=0$ is assumed to lie at this peak, $\lambda_{O}=\lambda_{\text {peak }}$. The wavelength of the other modes is $\lambda_{p}=\lambda_{0}+p \Delta \lambda$ where $\Delta \lambda$ is the mode wavelength separation. The second term $G_{N L(p)}$ is a nonlinear terms and describes suppression of the gain of an arbitrary mode $p$ due to the increase in the intensity of the mode itself and the intensities of the other oscillating modes [26, 35],

$$
G_{N L(p)}=B S_{p}+\sum_{q \neq p}\left[D_{p(q)}+H_{p(q)}\right] S_{q}
$$

where $B$ is the coefficient of self-mode gain suppression, and $S_{p}$ and $S_{q}$ are the photon number of modes $p$ and $q$, respectively. The second cross-modal gain term controls the spectral broadening of the modal gain. For arbitrary mode $p$, the term $D_{p(q)} S_{q}$ is called SGS and is characterized by the coefficient $D_{p(q)}$, which is spectrally symmetric around the central mode

$$
D_{p(q)}=\frac{4}{3} \frac{B}{\left(2 \pi c \tau_{\text {in }} / \lambda_{p}^{2}\right)\left(\lambda_{p}-\lambda_{q}\right)^{2}+1}
$$

where $c$ is the speed of light in free space and $\tau_{\text {in }}$ is the intraband relaxation time. This term then indicates equal gain suppression on the longer and shorter wavelength 
sides of the mode. The last term $H_{p(q)} S_{q}$ is called AGS and is characterized by the coefficient $H_{p(q)}$, which is spectrally asymmetric around the mode $p$,

$$
H_{p(q)}=\frac{3}{8 \pi}\left(\frac{a \xi}{V}\right)^{2}(N-\bar{N}) \frac{\alpha \lambda_{p}^{2}}{\lambda_{q}-\lambda_{p}}
$$

where $\bar{N}$ is the time-averaged value of $N$. This equation then indicates that $H_{p(q)}$ is positive and gain suppression becomes higher for modes on the longer wavelength side $\left(\lambda_{q}>\lambda_{p}\right)$ of the mode, whereas it is negative and gain suppression becomes lower for modes on the shorter wavelength side $\left(\lambda_{q}<\lambda_{p}\right) . H_{p(q)}$ is then enhanced not only for larger values of $\alpha$ but also for larger values of the gain spectrum width $b$ [27]. For lower values of $\alpha$ and/or $b$, the gain spectrum is almost homogeneous. Both experiment and theory reported on inhomogeneous, or specifically asymmetric broadening of modal gain in long-wavelength semiconductor lasers [23-26] because of the large values of the linewidth enhancement factor $\alpha$ [36].

The operation characteristics of the oscillating modes of semiconductor laser subject to optical feedback are obtained by numerical integration of the following timedelay rate equations of the injected carrier number $N(t)$, modal photon number $S_{p}(t)$ and phase $\theta_{p}(t)$ :

$$
\begin{aligned}
\frac{d N}{d t}= & \frac{1}{e} I(t)-\sum_{p} A_{P} S_{p}-\frac{N}{\tau_{s}} \\
\frac{d S_{p}}{d t}= & \left(G_{p}-G_{t h 0}+\frac{c}{n_{D} L_{D}} \ln \left|U_{p}(t-\tau)\right|\right) S_{p} \\
& +\frac{a \xi N / V}{4\left(\frac{\lambda_{p}-\lambda_{\text {pak }}}{\delta \lambda}\right)^{2}+1} \\
\frac{d \theta_{p}}{d t}= & \frac{1}{2}\left(\frac{\varepsilon a \xi}{2 V}(N-\bar{N})-\frac{c}{n_{D} L_{D}} \phi_{b}\right)
\end{aligned}
$$

where $G_{t h o}$ is the threshold gain of the solitary laser (without feedback) and $\delta \lambda$ is the full width at half maximum (FWHM) of the spontaneous emission spectrum. The optical feedback is included in terms of the timedelay function $U(t-\tau)$, which is a function of the power reflectivities $R_{f}$ and $R_{e x}$ of the front facet mirror and external reflector, respectively, as

$$
\begin{aligned}
U_{p}(t-\tau) & =\left|U_{p}\right| e^{-j \phi_{p}} \\
& =1-\frac{1-R_{f}}{R_{f}} \sum_{m=1}{\sqrt{R_{f} \eta R_{e x}}}^{m} e^{-j m \omega \tau} \sqrt{\frac{S_{p}(t-m \tau)}{S_{p}(t)}} \frac{e^{j \theta_{p}(t-m \tau)}}{e^{j \theta_{p}(t)} \text { That is the gain becomes }} \\
(9) \quad G_{p} & =A_{p}-B \sum_{p} S_{p}=A_{p}-B S
\end{aligned}
$$

This function considers optical feedback as a time delay of laser mode radiation due to multiple round trips in the external cavity. The period of each round trip is $\tau=2 n_{e x} L_{e x} / c$, where $n_{e x}$ and $L_{e x}$ are the refractive index and length of the external cavity, respectively. The rate eqs. (5)-(8) represent generalization of the LangKobayashi model [11] to the case of multimode laser with multiple round-trip feedback.

The fourth-order Runge-Kutta algorithm is applied to integrate rate eqs. (6)-(8) using a time step as shirt as $\Delta t=0.5 \mathrm{ps}$. Fifteen modes $(p=-7 \rightarrow+7)$ are considered in the calculations. The parameters of a quaternary 1550-nm InGaAsP laser coupled with a very-short external cavity used in calculations are listed in Table 1 . The injection current is set at $49 \mathrm{~mA}$, which is five times the threshold current. The length of the external cavity is as short as $3.5 \mathrm{~mm}$, which corresponds to round trip propagation delay time of $\tau=16.67 \mathrm{ps}$ in the feedback cavity. These parameters in the single-mode version are predicted to support intensity modulation up to frequencies as high as $55 \mathrm{GHz}$ [21]. The SMSR of the multimode oscillation is evaluated as the ratio of the average value of the power of the dominant mode to that of the strongest side mode. We decide the laser oscillation to be in the single mode operation when SMSR $=100$ or $20 \mathrm{~dB}$.

\section{Results}

First, we gain inside into the modal oscillations and the dominating multimode operation in the solitary laser. Figure 1a plots a typical output multimode spectrum of the InGaAsP laser with the Fabry-Perot structure. The gain spectrum is plotted on right-side axis. In this case the side mode suppression ratio is as small as SMSR = $S_{+1} / S_{+2}=1.4$, which is much smaller than the singlemode oscillation of SMSR $=100$. As the figure indicates, the spectrum is asymmetric around the most dominant mode $p=+1$ as a result of AGS in eq. (5). This asymmetric output spectrum is manifestation of the asymmetric gain profile shown in the same figure, and was observed in experiment [28]. AGS works to induce instantaneous mode hopping in the transient region among some modes $(p=0,+1$ and +2$)$ on the longerwavelength side of central mode whose total gain can exceed the linear gain of the central mode [26].

In order to clarify the influence of the AGS on the laser output, we plot in Fig. 1b the corresponding output spectrum for the case of HGB. In this case, the coefficients of cross-modal gain suppression become

where $S=\sum_{p} S_{p}$ is the total photon number. Therefore, the spectral characteristics of the gain are determined by 
Table 1 Typical values of InGaAsP multimode laser coupled with very-short external cavity used in the calculations

\begin{tabular}{|c|c|c|c|}
\hline Symbol & Definitions of parameters & Value & Unit \\
\hline $\bar{\lambda}$ & Wavelength & 1550 & $\mathrm{~nm}$ \\
\hline v & Active layer volume & $3 \times 10^{-11}$ & $\mathrm{~cm}^{3}$ \\
\hline$L_{D}$ & Length of active region & 120 & $\mu \mathrm{m}$ \\
\hline$n_{D}$ & Refractive index in active region & 3.6 & - \\
\hline$R_{f}$ & Power reflectivity at front facet & 0.2 & - \\
\hline$a$ & Differential gain coefficient & $8.24 \times 10^{-16}$ & $\mathrm{~cm}^{2}$ \\
\hline$N_{g}$ & Carrier density at transparency & $3.69 \times 10^{7}$ & - \\
\hline$\xi$ & Mode confinement factor & 0.15 & \\
\hline$\tau_{s}$ & Carrier lifetime & 776 & ps \\
\hline$\tau_{p}$ & Photon lifetime & 1.69 & ps \\
\hline$\tau_{\text {in }}$ & Intraband relaxation time & 0.1 & ps \\
\hline B & Self-modal gain compression coefficient & $9.23 \times 10^{-7}$ & $s^{-1}$ \\
\hline$b$ & Dispersion parameter of linear gain spectrum spectrumession coefficient & $5.0 \times 10^{37}$ & $m^{-3} A^{-2}$ \\
\hline$\delta \lambda$ & FWHM of spontaneous emission & 23 & $\mathrm{~nm}$ \\
\hline$L_{e x}$ & Length of external cavity & 3.5 & $\mathrm{~mm}$ \\
\hline$n_{e x}$ & Refractive index of external cavity & 1.0 & - \\
\hline
\end{tabular}

the linear gain and the oscillating modes are homogeneously suppressed. Although the figure indicates improvement of the SMSR under HGB to 14.8, this value is still smaller than 100 , and the laser oscillates in multimode. In this case, the central mode, $p=0$, maintains the highest

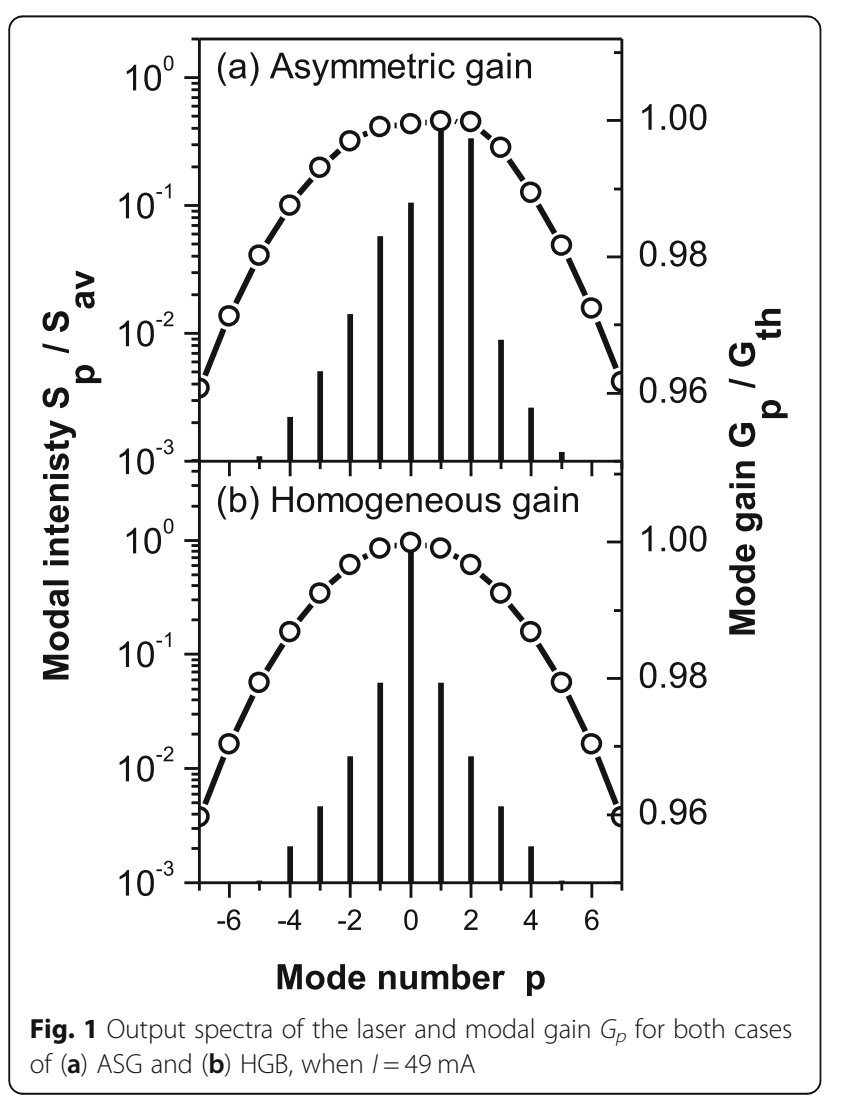

intensity as it has the highest gain as shown in the symmetric gain profile of Fig. 1b.

The influence of optical feedback from the very close reflector on the modal oscillation of the investigated laser is depicted in Fig. 2. The figure plots SMSR versus the external reflectivity $\Gamma R_{e x}$ (measure of the coupled light to the primary laser cavity) normalized by the power reflectivity $R_{f}$ at the front facet. It is worth noting that the laser operates in CW over the relevant range of $\Gamma R_{e x} / R_{f}$, which is a typical advantage of using the very short external cavity in avoiding the noisiest state of

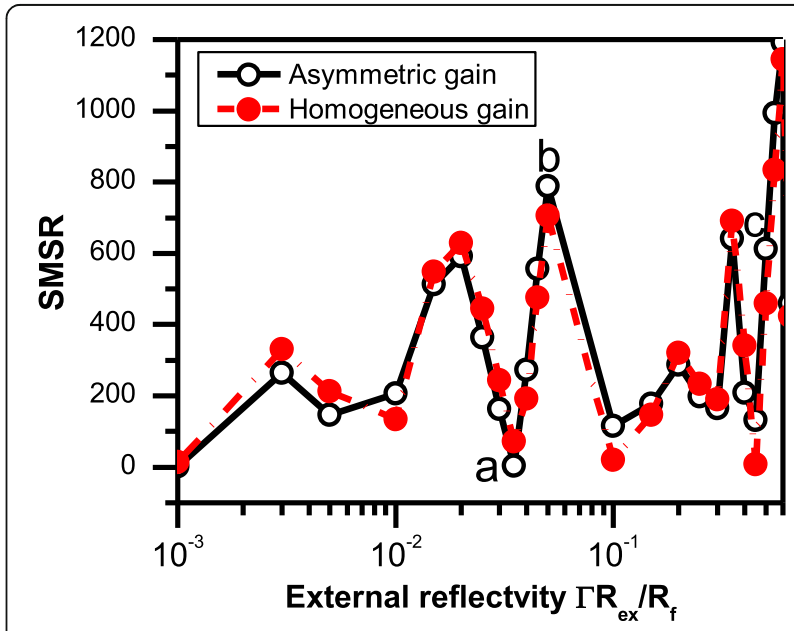

Fig. 2 SMSR of the semiconductor laser as function of the external power reflectivity for both cases of asymmetric gain and HGB 
chaos [20]. The figure shows that except for the narrow ranges of $\Gamma R_{e x} / R_{f}<0.002$ and $0.032 \leq \Gamma R_{e x} / R_{f} \leq 0.037$, and the laser oscillates predominantly in single mode with SMSR > 100, which is another advantage of using the very short external cavity to achieve the single-mode oscillation. The figure plots also the SMSR values when considering HGB, showing almost similar behavior to that under asymmetric gain with the variation of the feedback strength. That is, the influence of optical feedback on the modal oscillation in this case of very-short external cavity is independent of the spectral distribution of the optical gain. This result is confirmed by reporting that the predominantly oscillating mode jumps among the oscillating modes in the range of $-2 \leq p \leq+2$ for both cases of asymmetric gain and HGB. This conclusion agrees with the reports by Ahmed and Yamada [28[and Ahmed [29] that the mode competition phenomenon induces motion of the lasing mode to the long- or shortwavelength in the case of short external cavity.

In Fig. $3 \mathrm{a}-\mathrm{c}$, we characterize the mode oscillations at the three specific points $a, b$ and $c$, respectively of Fig. $2\left(\Gamma R_{e x} / R_{f}=0.035,0.05\right.$, and 0.5$)$ by plotting the corresponding output spectra. In Fig. 3a, the laser output is mainly contained in the two modes $p=0$ and +1 with the SMSR being improved to SMSR $=S_{+}$ ${ }_{1} / S_{+2}=2.86$. In Figs. 3b, the laser oscillates predominantly in the long-wavelength side mode $p=+1$, where SMSR jumps to the large value of SMSR $=788$. That is, the influence of AGS is still active to enhance the gain of mode $p=+1$ over the gain of the central mode $p=0$. In Fig. 3c of $\Gamma R_{e x} / R_{f}=0.5$, the laser still maintains single mode oscillation but in the central mode $p=0$ with large of value of $\operatorname{SMSR}=612$. This means that in this case, the feedback works against ASG and retrieves the gain mode $p=0$ at the expense of the gain of mode $p=+1$.

In the previous calculations, the external optical feedback was assumed similar for all oscillating modes, which corresponds to a plane external mirror with flat power reflectivity over the spectrum of the modal gain. From now on, we will consider narrow-band wavelengthselective optical feedback produced by an external Bragg grating with the following Lorentzian distribution [37].

$$
R_{e x(p)}=R_{\text {peak }}\left[\eta \frac{\Delta \lambda_{g}^{2}}{\Delta \lambda_{g}^{2}+\left(\lambda_{\text {peak }}-\lambda_{p}\right)^{2}}\right]^{2}
$$

where $R_{\operatorname{ex}(p)}$ is the power reflectivity of mode $p$ of wavelength $\lambda_{p} . \Delta \lambda_{g}$ is the grating bandwidth, $\lambda_{\text {peak }}$ and $R_{\text {peak }}$ are the peaked wavelength and power reflectivity, respectively, and $\eta$ is the diffraction efficiency of the grating. We use this form of the modal external reflectivity in eq. (9) of the time-delay feedback function $U_{p}(t-\tau)$ and detune the peak wavelength $\lambda_{\text {peak }}$ to fit with the wavelength of one of oscillating modes. We fix the power reflectivity $R_{\text {peak }}$ to correspond to one of the feedback levels $\Gamma R_{e x} / R_{f}$ that reveal single-mode oscillations as indicated in Fig. 3. Figure 4 depicts a bar plot of the intensity of the oscillating mode $p$ as a function of the detuning wavelength $\left(\lambda_{p}-\lambda_{\text {peak }}\right)$ of the investigated laser coupled with a cavity with selective feedback. The figure corresponds to $\Gamma R_{e x} / R_{f} \sim 0.05$ which corresponds to oscillation in mode $p=+1$, as shown in Fig. 2b. The figure shows that not only oscillation in single mode is achieved but also selective wavelength of this oscillation is obtained. The wavelength-tuning range is as wide as $19.7 \mathrm{~nm}$, which corresponds to span of the oscillating modes between $p=-3$ to $p=+4$. This range could be more or less depending on the level of optical feedback in either regimes of intermediate or strong feedback.

\section{Conclusions}

We presented the improvement of modal oscillation of longitudinal multimode semiconductor laser into oscillation in single mode by means of optical feedback from a very short external cavity. The study was based a timedelay rate equations of multimode semiconductor laser

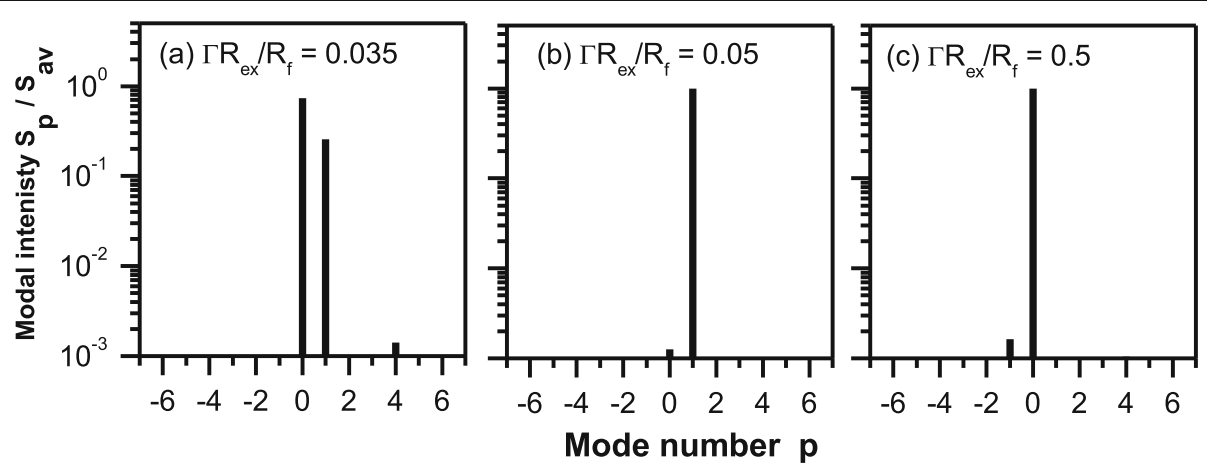

Fig. 3 Output spectra of the laser under OFB strengths of (a) $\Gamma R_{e x} / R_{f}=0.035$, (b) $\Gamma R_{e x} / R_{f}=0.05$, and (c) $\Gamma R_{e x} / R_{f}=0.5$ 


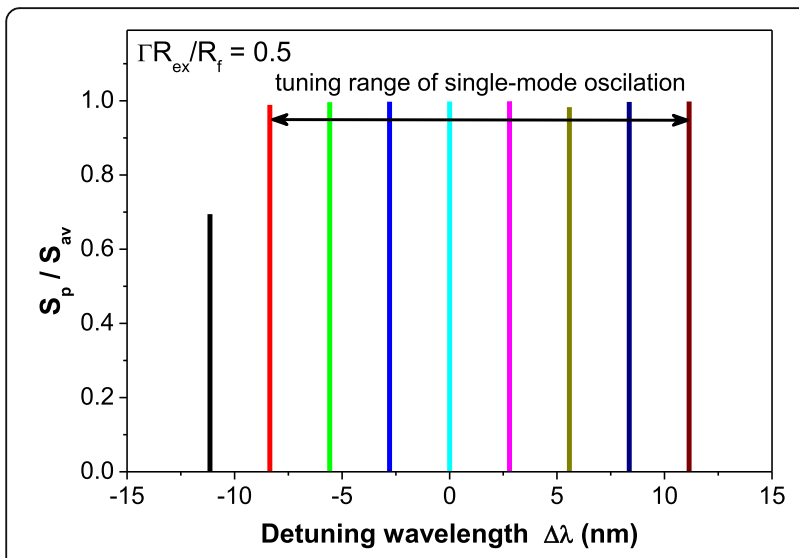

Fig. 4 Detuning output spectra of the laser as a function of the detuning wavelength $\Delta \lambda=\lambda_{p}-\lambda_{\text {peak }}$ under OFB strength of $\Gamma R_{e x} / R_{f}=0.5$

including the symmetric and asymmetric suppressions of modal gain. The obtained results showed that the optical feedback releases AGS and increases the gain of one of the oscillating mode close to the central mode, which induces single mode oscillation. In addition, by using selective optical feedback and detuning the peaked wavelength of feedback relative to the central wavelength of gain, the predominant mode can be tuned over a wide wavelength range of more or less than $20 \mathrm{~nm}$.

\section{Acknowledgements}

This work is funded by the Deanship of Scientific Research (DSR), King Abdulaziz University, Jeddah, under grant no. (RG-9-130-38). The authors, therefore, acknowledge with thanks DSR technical and financial support.

\section{Authors' contributions}

All authors contribute to the modeling, simulation, discussion and preparation of the manuscript. All authors read and approved the final manuscript.

\section{Funding}

This work is funded by the Deanship of Scientific Research (DSR), King Abdulaziz University, Jeddah, under grant no. (RG-9-130-38).

\section{Availability of data and materials}

Please contact authors for data requests.

\section{Competing interests}

The authors declare that they have no competing interests.

Received: 17 June 2019 Accepted: 16 September 2019 Published online: 16 October 2019

\section{References}

1. Lenstra, D., Verbeek, B.H., den Boef, A.J.: Coherence collapse in single-mode semiconductor lasers due to optical feedback. IEEE J. Quantum Electron. QE-21, 674-679 (1985)

2. Schunk, N., Petermann, K: Numerical analysis of the feedback regimes for a single-mode semiconductor laser with external feedback. IEEE J. Quantum Electron. 24, 1242-1247 (1988)

3. Tromborg, B., Mork, J.: Stability analysis and the route to chaos for laser diodes with optical feedback. IEEE Photon. Technol. Lett. 2, 549-552 (1990)

4. Mork, J., Tromborg, N., Mork, J.: Chaos in semiconductor lasers with optical feedback: theory and experiment. IEEE J. Quantum Electron. 28 93-108 (1992)
5. Abdulrhmann, S., Ahmed, M., Okamoto, T., Ishimori, W., Yamada, M.: An improved analysis of semiconductor laser dynamics under strong optical feedback. IEEE J. of Sel. Top. Quantum Electron. 9, 1265-1274 (2003)

6. Ahmed, M., Yamada, M.: Field fluctuations and spectral lineshape in semiconductor lasers subjected to optical feedback. J. Appl. Phys. 95, 75737583 (2004)

7. Mork, J., Mark, J., Tromborg, B.: Route to chaos and competition between relaxation oscillations for a semiconductor laser with optical feedback. Phys. Rev. Lett. 65, 1999-2002 (1990)

8. Mork, J.: Nonlinear Dynamics and Stochastic Behaviour of Semiconductor LasersPhD thesis, Rep. S48, Technical University of Denmark (1989)

9. Schunk, N., Petermann, K.: Stability analysis for laser diodes with short external cavities. IEEE Photon. Technol. Lett. 1, 49-51 (1989)

10. Helms, J., Petermann, K.: A simple analytic expression for the stable operation range of laser diodes with optical feedback. IEEE J. Quant. Electron. 26, 833-836 (1990)

11. Lang, R., Kobayashi, K.: External optical feedback effects on semicobductor laser properties. IEEE J. Quantum Electron. QE 16, 347-355 (1980)

12. Tager, A.A., Petermann, K.: High frequency oscillations and self-mode locking in short external cavity laser diodes. IEEE J. Quant. Electron. 30, 1553-1561 (1994)

13. Ryan, A.T., Agrawal, G.P., Gray, G.R., Gage, E.C.: Optical-feedback-induced chaos and its control in multimode semiconductor lasers. IEEE J. Quant. Electron. 30, 668-678 (1994)

14. Petermann, K.: External optical feedback phenomena in semiconductor lasers. IEEE J. Quant. Electron. 1, 480-489 (1995)

15. Tkach, E.W., Chraplyvy, A.R.: Regimes of feedback effects in 1.5-mm distributed feedback lasers. IEEE J. Lightwave Technol. LT-4, 1655-1961 (1986)

16. Lenstra, D.: Statistical theory of the multistable external-feedback laser. Opt. Commun. 81, 209-214 (1991)

17. Tromborg, B., Osmundsen, J.H., Olesen, H.: Stability analysis for a semiconductor laser in an external cavity. IEEE J. Quant. Electron. 20 1023-1032 (1984)

18. Kao, Y.H., Wang, N.M., Chen, H.M.: Mode description of routes to chaos in external-cavity coupled semiconductor lasers. IEEE J. Quantum Electron. 30, 1732-1739 (1994)

19. Ahmed, M., Yamada, M., Abdulrhmann, S.: Numerical modeling of the route-to-chaos of semiconductor lasers under optical feedback and its dependence on the external-cavity length. Intl. J. Numer. Model. 22, 434-445 (2009)

20. Jones, R.J., Spencer, P.S., Lawrence, J., Kane, D.M.: Influence of external cavity length on the coherence collapse regime in laser diodes subject to optical feedback. IEE Proc. Optoelectron. 148, 7-12 (2001)

21. Ahmed, M., Bakry, A., Altuwirgi, R., Alghamdi, M., Koyama, F.: Enhancing modulation bandwidth of semiconductor lasers beyond $50 \mathrm{GHz}$ by strong optical feedback for use in mm-wave radio over fiber links. Jpn. J. Appl. Phys. Rap. Comm. 53, 124103 (2013)

22. Liu, P., Ogawa, K.: Statistical measurements as a way to study mode partition in injection lasers. J. Lightwave Technol. LT-2, 44-48 (1984)

23. Ogita, S., Lowery, A.J., Tucker, R.S.: Influence of asymmetric nonlinear gain on the transient of longitudinal modes in long wavelength Fabry-Perot laser diodes. IEEE J. Quantum Electron. 33, 198-210 (1997)

24. Ogasawara, N., Ito, R.: Longitudinal mode competition and asymmetric gain saturation in semiconductor injection lasers: I. experiment. Jpn. J. Appl. Phys. 27, 607-614 (1988)

25. Ogasawara, N., Ito, R.: Longitudinal mode competition and asymmetric gain saturation in semiconductor injection lasers: II. Theory. Jpn. J. Appl. Phys. 27, 615-626 (1989)

26. Ahmed, M., Yamada, M.: Influence of instantaneous mode-competition on the dynamics of semiconductor lasers. IEEE J. Quantum Electron. 38, 682-693 (2002)

27. Yamada, M., Ishimori, W., Sakaguchi, H., Ahmed, M.: Time dependent measurement of the mode competitlon phenomena among longitudinal modes in long-wavelength lasers,' IEEE J. Quantum Electron. 39, 1548-1554 (2003)

28. Ahmed, M., Yamada, M.: Inducing single-mode oscillation in Fabry-Perot InGaAsP lasers by applying external optical feedback. IET Optoelectron. 4, 133-141 (2010)

29. Ahmed, M.: Longitudinal-mode competition in semiconductor lasers under external optical feedback: regime of short cavity. Opt. Laser Technol. 41, 53-63 (2009) 
30. Green, P.E.: Fibre Optic Networks. Prentice Hall, Englewood Cliffs, NJ (1993)

31. Wiemann, C., Hollberg, L.: Using diode lasers for atomic physics. Rev. Sci. Instrum. 62, 1-20 (1990)

32. Lohman, A., Syms, R.R.A.: External cavity laser with a vertically etched silicon blazed grating. IEEE Photonic. Techn. L. 15, 120-122 (2003)

33. Rosenblatt, D., Sharon, A., Friesem, A.A.: Resonant grating waveguide structures. IEEE J. Quantum Elect. 33, 2038-2205 (1997)

34. Block, S., Gamet, E., Pigeon, F.: Semiconductor laser with external resonant grating mirror. IEEE J. Quantum Elect. 41, 1049-1053 (2005)

35. Yamada, M.: Theoretical analysis of nonlinear optical phenomena taking into account the beating vibration of the electron density in semiconductor lasers. J. Appl. Phys. 66, 81-89 (1989)

36. Osinski, M., Buus, J.: Linewidth broadening factor in semiconductor lasers-an overview. IEEE J. Quantum Electron. QE-23, 9-29 (1987)

37. Hernandez, G.: Fabry-Perot Interferometers. Cambridge University Press, Cambridge (1986)

\section{Publisher's Note}

Springer Nature remains neutral with regard to jurisdictional claims in published maps and institutional affiliations.

\section{Submit your manuscript to a SpringerOpen ${ }^{\circ}$ journal and benefit from:}

- Convenient online submission

- Rigorous peer review

- Open access: articles freely available online

- High visibility within the field

- Retaining the copyright to your article

Submit your next manuscript at $\boldsymbol{\wedge}$ springeropen.com 